International Journal of Pharmaceutics 391 (2010) 212-220

\title{
Improved plasma stability and sustained release profile of gemcitabine via polypeptide conjugation
}

\author{
Lik-Voon Kiew $^{\mathrm{a}}$, Soon-Keng Cheong ${ }^{\mathrm{b}}$, Khalifah Sidik ${ }^{\mathrm{c}}$, Lip-Yong Chung ${ }^{\mathrm{d}}$ \\ ${ }^{a}$ Department of Pharmacology, Faculty of Medicine, University of Malaya, 50603 Kuala Lumpur, Malaysia \\ ' IMU Clinical School, International Medical University, 70300 Seremban, Malaysia \\ ${ }^{\mathrm{C} D e p a r t m e n t}$ of Biomedical Sciences, University of Illinois College of Medicine, Rockford, IL 61107, USA \\ ${ }^{\mathrm{d} D e p a r t m e n t}$ of Pharmacy, Faculty of Medicine, University of Malaya, 50603 Kuala Lumpur, Malaysia
}

\begin{abstract}
To enhance the stability of the anticancer drug gemcitabine (2'-deoxy-2',2'-difluorocytidine), it was conjugated to poly-I-glutamic acid (PG-H) via a carbodiimide reaction. The synthesised poly-I-glutamic acid-gemcitabine (PG-G) was purified and characterised by using SDS-PAGE to estimate its molecular weight, HPLC to determine its purity and degree of drug loading, and NMR to elucidate the structure. In vitro aqueous hydrolytic studies showed that the gemcitabine release from the polymeric drug conjugate was $\mathrm{pH}$ dependent, and that the conjugation to PG-H improved its stability in human plasma. The release of the bound gemcitabine from PG-G in plasma was mediated by a hydrolytic process. It began with a lag phase, followed by linear release between 12 and $48 \mathrm{~h}$, and reached equilibrium at $72 \mathrm{~h}$ with $51 \%$ of the gemcitabine released. In vitro cytotoxicity studies using MCF-7 and MDA-MB-231 human mammary cancer cells, as well as human dermal fibroblasts (HDF), showed that PG-G displayed a lower dose dependent cytotoxic effect with respect to the parent drug gemcitabine. On the other hand, in 4T1 mouse mammary tumour cells, PG-G and gemcitabine showed similar toxicities. Gemcitabine was more than likely released hydrolytically from PG-G and taken up by MCF-7, MDA-MB-231 and HDF, whereas both released gemcitabine and PG-G were taken up by $4 \mathrm{~T} 1$ to mediate the observed cytotoxicities. The improved stability and extended sustained release profile may render PG-G a potential anticancer prodrug.
\end{abstract}

(Received 18 November 2009, Received in revised form 31 December 2009, Accepted 2 March 2010, Available online 7 March 2010) 


\section{Keywords:}

Animals, Cell Line, Tumor, Cell Survival/drug effects, Delayed-Action Preparations, Deoxycytidine/administration \& dosage/*analogs \& derivatives/blood/chemical synthesis/chemistry/pharmacokinetics/pharmacology, Drug Screening Assays, Antitumor, Drug Stability, Female, Humans, Polyglutamic Acid/administration \& dosage/*analogs \& derivatives/blood/chemical synthesis/pharmacokinetics/pharmacology, Prodrugs/administration \& dosage/*chemical synthesis/*pharmacokinetics/pharmacology

DOI: 10.1016/j.ijpharm.2010.03.010

URL:

http://www.ncbi.nlm.nih.gov/pubmed/20214970

http://www.sciencedirect.com/science/article/pii/S0378517310001845

http://pages.aaps.org/elsevier/view.asp?ID=119519

http://cat.inist.fr/?aModele $=$ afficheN\&cpsidt=22753637

http://d.wanfangdata.com.cn/NSTLQK_10.1016-j.ijpharm.2010.03.010.aspx

\section{Suggested citation format:}

Kiew LV, Cheong SK, Sidik K, Chung LY. Improved plasma stability and sustained release profile of gemcitabine via polypeptide conjugation. International journal of pharmaceutics. 2010;391(1-2):212-20 